\title{
Ultrasound measurements of gastrocnemius muscle thickness in older people with sarcopenia
}

This article was published in the following Dove Press journal:

Clinical Interventions in Aging

Jing Wang

Ying $\mathrm{Hu}$

Guo Tian

Department of Ultrasound, The First Affiliated Hospital, Medical College,

Zhejiang University, Hangzhou,

Zhejiang 310003, China
Correspondence: Jing Wang Department of Ultrasound, The First Affiliated Hospital, Medical College, Zhejiang University, 79 Qingchun Road, Hangzhou, Zhejiang 310003, China Tel/fax +86 57l 87236628

Email yanshu006@zju.edu.cn
Background: Sarcopenia, defined as low muscle mass and low muscle strength and/or low physical performance, is affecting more and more people. The European Working Group on Sarcopenia in Older People has suggested the routine community screening. However, selecting the most suitable method to evaluate muscle mass to detect sarcopenia in community screening is a challenge. This study sought to analyze the correlation between ultrasound (US) measurements of the gastrocnemius muscle and low muscle mass, as defined by sarcopenia.

Methods: One hundred thirty-five elderly participants were enrolled. US measurements included muscle thickness (MT), fat thickness (FT), MT/body mass index (BMI), and MT/FT. The definition of low muscle mass was based on the proposal from the Asian Working Group for Sarcopenia, in which the cutoff values for low muscle mass were $7.0 \mathrm{~kg} / \mathrm{m}^{2}$ for men and $5.4 \mathrm{~kg} / \mathrm{m}^{2}$ for women using dual energy X-ray absorptiometry (DXA). The participants were divided into low and normal muscle mass groups. Participants with low muscle mass were divided into presarcopenia, sarcopenia, and severe sarcopenia groups.

Results: Those in the low muscle mass group were older and had lower weights and BMIs $(P<0.05)$. In addition, MT and MT/BMI were lower in the low muscle mass group $(P<0.05)$. Binary logistic regression analysis revealed that MT was the factor associated with low skeletal muscle mass $(\mathrm{OR}=0.001, P<0.001)$. ANOVA revealed that $\mathrm{MT}$ was not significantly different between subjects with presarcopenia, sarcopenia, and severe sarcopenia ( $F=1.69, P=0.192)$. Receiver operating characteristic curve analyses indicated that the cutoff value of the gastrocnemius MT for low muscle mass was $1.50 \mathrm{~cm}$.

Conclusion: MT measured by US was identified as the factor associated with low skeletal muscle mass. Those with gastrocnemius MT less than $1.5 \mathrm{~cm}$ can be considered as low muscle mass.

Keywords: ultrasound, muscle mass, sarcopenia, gastrocnemius muscle

\section{Introduction}

Sarcopenia is defined as a geriatric syndrome characterized by progressive and generalized loss of skeletal muscle mass and muscle function (muscle strength and/or physical performance) with a risk of adverse outcomes, such as physical disability, falls, poor quality of life, and death. ${ }^{1-3}$ It has been reported that sarcopenia is related to obesity, metabolic syndrome, blood sugar abnormality, cardiovascular disease, osteoporosis, fracture, cognitive impairment, and depression. ${ }^{4-6}$ The diagnosis of sarcopenia requires documentation of muscle mass and documentation of muscle strength and/or physical performance. ${ }^{7-10}$ The European Working Group on Sarcopenia in Older People (EWGSOP) has suggested a conceptual staging system that progresses from "presarcopenia" (low muscle mass only) and "sarcopenia" (low muscle mass with low muscle strength or low physical performance) to "severe sarcopenia" (low muscle mass with low muscle strength and low physical performance). ${ }^{7}$ Currently, sarcopenia affects more 
than 50 million people and will affect more than 200 million people in the next 40 years. $^{7}$ Thus, the EWGSOP suggests that elderly people should be screened routinely in communities. ${ }^{7}$ At present, consensus statements from different regions around the world generally suggest using dual energy X-ray absorptiometry (DXA) and bioelectrical impedance analysis (BIA) to gage muscle mass, grip strength to gage muscle strength, and gait speed to gage physical performance. In fact, there are several methods to measure muscle mass, such as anthropometry, 24-hour creatinine measurement, ultrasound (US), computed tomography (CT), magnetic resonance imaging (MRI), DXA, and BIA. MRI and CT are known as the gold standards for measuring skeletal muscle mass and have been used primarily for basic or clinical research studies. However, these methods are limited because they are expensive and not portable and CT involves exposure to radiation. DXA and BIA have been widely and commonly used in the diagnosis of sarcopenia. However, the results form BIA can be affected by the formula of the instrument, the measurement environment, and the condition of the skin, as well as the alterations in hydration status. ${ }^{11}$ Despite the minimal radiation exposure from DXA, it is difficult to use in community-wide screenings of sarcopenia. In addition, DXA is not portable. Moreover, other methods of predicting skeletal muscle mass show poor accuracy.

Thus, selecting the most suitable method for evaluating muscle mass in a community setting is a challenge. Since most of the skeletal muscle in the body exists in the limbs, the amount of muscle in the limbs represents the overall muscle mass. ${ }^{12}$ It was indicated that the US prediction equation is a valid method to predict skeletal muscle mass and represents an alternative to MRI measurement. ${ }^{13} \mathrm{US}$ is portable and has the advantage of rapid measurement time. Furthermore, the skeletal muscle mass measured by US has been shown to be suitable. ${ }^{14}$

The primary purpose of the present study was to analyze the correlation between skeletal muscle mass and skeletal muscle measurements obtained by US and to select appropriate diagnostic cutoff values of US measurements for low muscle mass, as defined by sarcopenia.

\section{Methods}

\section{Participants}

In this cross-sectional study, 183 participants (50 males and 133 females, aged $\geq 60$ years) who were admitted to a community health examination in Health Management Center, The First Affiliated Hospital, Medical College,
Zhejiang University, Hangzhou, Zhejiang, China, were enrolled. All participants were evaluated, including medical histories and clinical examinations. Participants with severe liver disease, kidney disease, diabetes, obesity, cardiovascular disease, cancer, and neuromuscular diseases, such as limb fracture, joint ligament injury, acute/chronic pain, cognitive impairment, and dementia, were excluded from this study, as were those who were taking drugs affecting metabolism, such as corticosteroids, estrogen, and thyroid hormone. A total of 48 (26.23\%) participants were excluded. Twentyfive participants temporarily refused to undergo DXA examination because of radiation exposure, and twenty-three participants were unable to perform the routine US position. Finally, 135 participants (39 males and 96 females, aged $71.25 \pm 7.64$ years) were included in this study.

All the participants were informed about the study procedure, and they provided a written informed consent before participating in the study. This study was approved by the ethics committee of The First Affiliated Hospital of Zhejiang University, Hangzhou, Zhejiang, China.

\section{US measurements}

Skeletal muscle measurements were done for the gastrocnemius muscle. The participants lay in the prone position with their legs extended and relaxed and with their feet hanging off the examination bed. The examiner held the US probe (2-10 MHz, Aixplorer; Aix-en-Provence, France) close to the skin, with the beam perpendicular to the surface of the skin, then probed the medial cross section of the medial head of the gastrocnemius muscle, and found the largest cross-sectional area as the standard section, marking the corresponding body surface. US measurements included fat thickness (FT, distance between the upper aponeurosis and skin) and muscle thickness (MT, distance between the upper and deeper aponeurosis) on the axial view (Figure 1), which were measured to the nearest $0.01 \mathrm{~cm}$. All the measurements were completed in the right lower limb. The participants were asked to take rest for 20 minutes after the first US measurements. The second US measurements were then taken in the marked point of the right lower limb. The final result was the average of the two measurements. The US measurements were carried out by one examiner (with 10 years of experience) who was blinded to the study results.

\section{Anthropometry parameters}

The subjects' current weight, height, and body mass index (BMI) were measured. Participants were measured in a room 


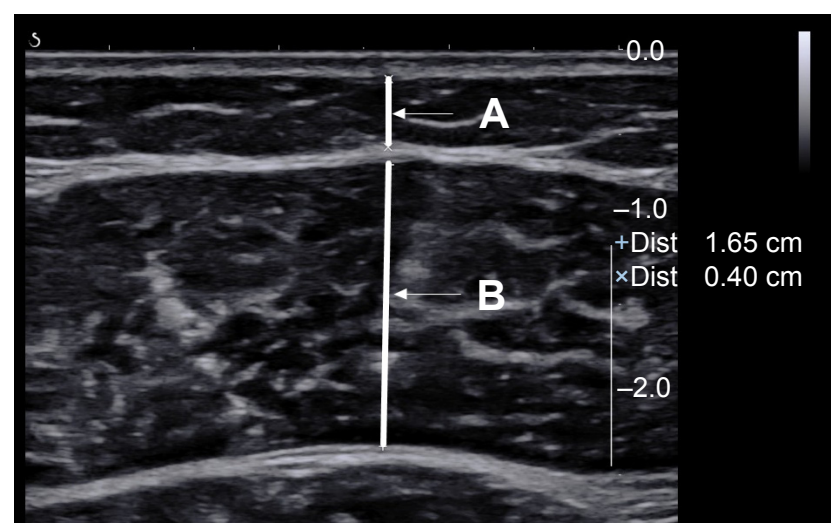

Figure I US imaging of the gastrocnemius muscle.

Note: FT (line A) and MT (line B) are illustrated on the axial view.

Abbreviations: FT, fat thickness; MT, muscle thickness; US, ultrasound.

with a temperature of $25^{\circ} \mathrm{C}$ and were required to take off the heavy coats. These parameters were measured using an US examination machine (SK-CK/TCS-160D-W/H; Shuangjia, Shenzhen, China). Body weight and height were measured to the nearest $0.01 \mathrm{~kg}$ and $0.01 \mathrm{~m}$, respectively. BMI was calculated as weight $(\mathrm{kg})$ divided by height $\mathrm{t}^{2}\left(\mathrm{~m}^{2}\right)$.

\section{Analysis indicators}

Herein, we used the BMI-adjusted definition of MT/BMI and the FT-adjusted definition of MT/FT. Finally, the analysis indicators in this study were as follows: gender (male and female), age (years), weight $(\mathrm{kg})$, height $(\mathrm{m})$, BMI $\left(\mathrm{kg} / \mathrm{m}^{2}\right)$, FT $(\mathrm{cm})$, MT $(\mathrm{cm})$, MT/BMI $\left(\mathrm{cm} / \mathrm{kg} / \mathrm{m}^{2}\right)$, and $\mathrm{MT} / \mathrm{FT}(\mathrm{cm} / \mathrm{cm})$.

\section{Muscle mass}

Participants underwent whole-body DXA (DISCOVERY-W fan-beam densitometer; Hologic Inc., Waltham, MA, USA), which can distinguish between three body components (fat mass, bone minerals, and fat-free soft tissue) using different X-ray attenuation properties. DXA was used to evaluate skeletal muscle mass. A height-adjusted definition of muscle mass/height ${ }^{2}$ was used, and the result was recorded in kilogram per meter square.

\section{Muscle strength}

Muscle strength was assessed by measuring grip strength using a JAMAR hand dynamometer (Asimow Engineering, Los Angeles, CA, USA). Participants were seated with their shoulders in an anatomical position and their elbows at $90^{\circ}$ flexion. The participants were shown how to use the dynamometer and were then asked to press on the grip for
5 seconds using their greatest possible force. The measurement was repeated after 1 minute. Both hands were measured separately, and the highest score was registered. The mean values of three trials were recorded in kilogram.

\section{Physical performance}

Physical performance was assessed by measuring gait speed with the 6-minute walk test. All the subjects were asked to walk at their normal gait speed. Gait speed was calculated as the average of two measurements and was recorded in meter per second.

\section{Diagnostic criteria}

The definition of sarcopenia was based on the proposal from the Asian Working Group for Sarcopenia (AWGS). ${ }^{9}$ The recommended cutoff values for low muscle mass were $7.0 \mathrm{~kg} / \mathrm{m}^{2}$ for men and $5.4 \mathrm{~kg} / \mathrm{m}^{2}$ for women using DXA. The recommended cutoff values for weak hand grip strength were $26 \mathrm{~kg}$ for men and $18 \mathrm{~kg}$ for women. The cutoff value for slow gait speed was $0.8 \mathrm{~m} / \mathrm{s}$.

According to the proposal from the EWGSOP, all the participants were divided into the following four groups: normal, presarcopenia, sarcopenia, and severe sarcopenia. In this study, the normal group included individuals with normal muscle mass, while the low muscle mass group included individuals with presarcopenia, sarcopenia, and severe sarcopenia.

\section{Statistical analyses}

To compare the characteristics of the normal and low muscle mass groups, such as age, weight, height, BMI, FT, MT, MT/BMI, and MT/FT, an independent $t$-test was performed and the results are represented as the mean $\pm \mathrm{SD}$. A chi-squared test was also performed to assess the effects of gender.

Binary logistic regression was performed in order to detect the potential variables for low muscle mass, results of which were shown using the ORs and their 95\% CI.

ANOVA was used for categorical comparisons to analyze the differences between the presarcopenia, sarcopenia, and severe sarcopenia groups.

The potential variables for low muscle mass were then analyzed using the receiver operating characteristic (ROC) curve analysis. When a significant cutoff value was observed, the sensitivity, specificity, and area under the curve (AUC) were presented.

Statistical analyses were performed using SPSS Version 17.0 for Windows software (SPSS Inc., Chicago, IL, USA). 
The two-sided values of $P<0.05$ were considered statistically significant.

\section{Results}

There were 76 participants who have low muscle mass suffering from sarcopenia among 135 participants. Participants in the low muscle mass group were older and had a lower body weight and BMI $(P<0.05)$. The US measurements, such as MT and MT/BMI, were lower in the low muscle mass group than those in the normal muscle mass group $(P<0.05)$. However, gender, height, FT, and MT/FT were not significantly different between the two groups (Table 1).

Table 2 shows the results of the binary logistic regression analysis evaluating the factor associated with low skeletal muscle mass. MT was the only significant factor associated with low muscle mass $(\mathrm{OR}=0.001, P<0.001)$.

The low muscle mass group $(n=76)$ included participants with presarcopenia $(n=57)$, sarcopenia $(n=14)$, and severe sarcopenia $(n=5)$. The MT in each group is shown in Table 3. MT was used as the test variable in the ANOVA, and the results indicated no significant difference between the presarcopenia, sarcopenia, and severe sarcopenia groups ( $F=1.69, P=0.192)$.

ROC curve analyses are shown in Figure 2. The sensitivity and specificity were 0.70 and 0.76 , respectively, and AUC was 0.82 , when the cutoff of MT for low muscle mass was $1.50 \mathrm{~cm}(P<0.001)$.

\section{Discussion}

Brodie $^{15}$ indicated that US is widely used for evaluating human body composition. There have been many reports of US measurements of skeletal muscle, such as skeletal muscle cross-sectional area, skeletal MT, FT, pennation angle, fascicle length, and echo intensity, among others. Jones et al ${ }^{16}$ investigated 24 adult cadavers with depth gage measurements of adipose to establish the validity between measurements of adipose thickness using US and radiography. The results showed a very close agreement between the US and radiography measurements on cadavers. Studies reported earlier also revealed the strong precision of US measurements for trunk and appendicular skeletal MT. ${ }^{17,18}$ It had been reported a significant and high correlation between skeletal muscle cross-sectional area and MT. ${ }^{19}$ Additionally, the pennation angle and fascicle length were confirmed to be the pertinent parameters measured to assess muscle mass. ${ }^{20,21}$ Some studies have reported changes in skeletal muscle with aging, such as increased intramuscular fat and altered echo intensity. ${ }^{22-24}$ Thus, the expected US measurements investigated in the current study included MT, FT, skeletal muscle cross-sectional area, muscle fascicle length, pennation angle, and echo intensity in muscle. Unfortunately, it was difficult to show all the participants' comprehensive imaging in one US screen, which might lead to inaccurate measurements of the cross-sectional area and fascicle length. The measurement of pennation angle was related to examiner-dependent techniques, as pennation angles were strongly influenced by the adjustment of the US probe. ${ }^{19}$ The measurement of echo intensity was affected by depth. In addition, it was impossible to compare the echo intensity values with those obtained using different devices because of the lack of a clear criterion for the echo intensity value. The US measurements of skeletal muscle above might be unsuitable for the routine community screening. Fortunately, the corrected limb measurement has been shown to be a reliable and accurate method

Table I Baseline characteristics of the normal and low muscle mass groups

\begin{tabular}{|l|l|l|l|l|}
\hline Variables & $\begin{array}{l}\text { Normal muscle mass } \\
(\mathbf{n}=\mathbf{5 9}), \text { mean } \pm \text { SD }\end{array}$ & $\begin{array}{l}\text { Low muscle mass } \\
(\mathbf{n}=\mathbf{7 6}), \text { mean } \pm \text { SD }\end{array}$ & $\chi^{2}$ or $\mathbf{P}$ & $P$-value \\
\hline $\begin{array}{l}\text { Gender }(\mathrm{n}) \\
\text { Male/female }\end{array}$ & $17 / 42$ & $22 / 54$ & $<0.00 \mathrm{I}$ & 0.986 \\
\hline Age $($ years $)$ & $69.34 \pm 7.06$ & $73.20 \pm 8.08$ & -2.96 & 0.004 \\
\hline Weight $(\mathrm{kg})$ & $64.97 \pm 10.23$ & $58.94 \pm 10.19$ & 3.40 & 0.001 \\
\hline Height $(\mathrm{m})$ & $1.61 \pm 0.07$ & $1.59 \pm 0.09$ & 1.13 & 0.260 \\
\hline BMI $\left(\mathrm{kg} / \mathrm{m}^{2}\right)$ & $24.99 \pm 2.78$ & $23.10 \pm 2.65$ & 3.99 & $<0.001$ \\
\hline FT $(\mathrm{cm})$ & $0.46 \pm 0.17$ & $0.43 \pm 0.17$ & 0.87 & 0.387 \\
\hline MT $(\mathrm{cm})$ & $1.63 \pm 0.23$ & $1.37 \pm 0.18$ & 7.28 & $<0.001$ \\
\hline MT/BMI $\left(\mathrm{cm} / \mathrm{kg} / \mathrm{m}^{2}\right)$ & $0.07 \pm 0.01$ & $0.06 \pm 0.01$ & 4.06 & $<0.001$ \\
\hline MT/FT $(\mathrm{cm} / \mathrm{cm})$ & $4.44 \pm 2.81$ & $3.81 \pm 1.86$ & 1.48 & 0.142 \\
\hline
\end{tabular}

Notes: An independent $t$-test was performed in age, weight, height, BMI, FT, MT, MT/BMI, and MT/FT. Chi-squared test was performed in gender. Age, weight, BMI, MT, and MT/BMI were significantly different between the normal muscle mass and low muscle mass groups $(P<0.05)$.

Abbreviations: BMI, body mass index; FT, fat thickness; MT, muscle thickness. 
Table 2 Binary logistic regression analysis of the muscle mass model

\begin{tabular}{|l|l|l|l|l|l|}
\hline Variables & B & Wald & P-value & OR & 95\% Cl \\
\hline MT & -7.15 & 26.65 & $<0.001$ & 0.001 & $0-0.01$ \\
\hline
\end{tabular}

Notes: The dependent variable was muscle mass. The independent variables included age, weight, BMI, MT, and MT/BMI. Low muscle mass was attributed to a value of I, and normal muscle mass was attributed to a value of 0 . MT was the only factor associated with low muscle mass.

Abbreviations: BMl, body mass index; MT, muscle thickness.

for estimating skeletal MT. A review analyzed 17 literature, and $69.23 \%$ of the literature studied muscle mass in the elderly by measuring MT with US. ${ }^{25}$ All told, the relevant US measurements among the analysis indicators in this study included FT, MT, MT/BMI, and MT/FT.

The gastrocnemius muscle was evaluated in this study for comparison to commonly applied/suggested anthropometric parameters for predicting sarcopenia. ${ }^{26}$ Moreover, Jones et $\mathrm{al}^{16}$ reported agreement between US and radiography measurements by measuring the calf $(r=0.98)$.

Our study divided participants into normal and low muscle mass groups. Those with low muscle mass were older and lighter in weight and had thinner muscles, including the MT/BMI. These results are in agreement with previous reports. ${ }^{26,27}$ However, according to the binary logistic regression results, there was only one significant factor associated with low muscle mass. The result obtained herein is similar to the studies before, in which the participants were divided into sarcopenia and nonsarcopenia groups. ${ }^{26,27}$ Thinner MT might be caused by the loss of muscle mass with aging.

Considering the existence of low muscle mass in all groups (presarcopenia, sarcopenia, and severe sarcopenia), the presence of differences in MT between the three stages is another question. Morat et al reported that MT was not significantly different between three different sarcopenia groups, which is in line with the development of the sarcopenia definition over the last several years. In this study, the results were in agreement with these findings and muscle strength and physical performance might play more important roles in different stages. ${ }^{11}$ In addition, Abe et a $\mathrm{a}^{17}$ reported

Table 3 MT in the presarcopenia, sarcopenia, and severe sarcopenia groups, given as the mean and SD

\begin{tabular}{|l|l|l|l|}
\hline Variables & Mean & SD & $95 \% \mathbf{C l}$ \\
\hline Presarcopenia $(n=57)$ & 1.39 & 0.19 & $1.34-1.44$ \\
\hline Sarcopenia $(n=14)$ & 1.33 & 0.09 & $1.27-1.38$ \\
\hline Severe sarcopenia $(n=5)$ & 1.26 & 0.28 & $0.91-1.60$ \\
\hline
\end{tabular}

Note: ANOVA was performed, with $F=1.69$ and $P=0.192$.

Abbreviation: MT, muscle thickness.

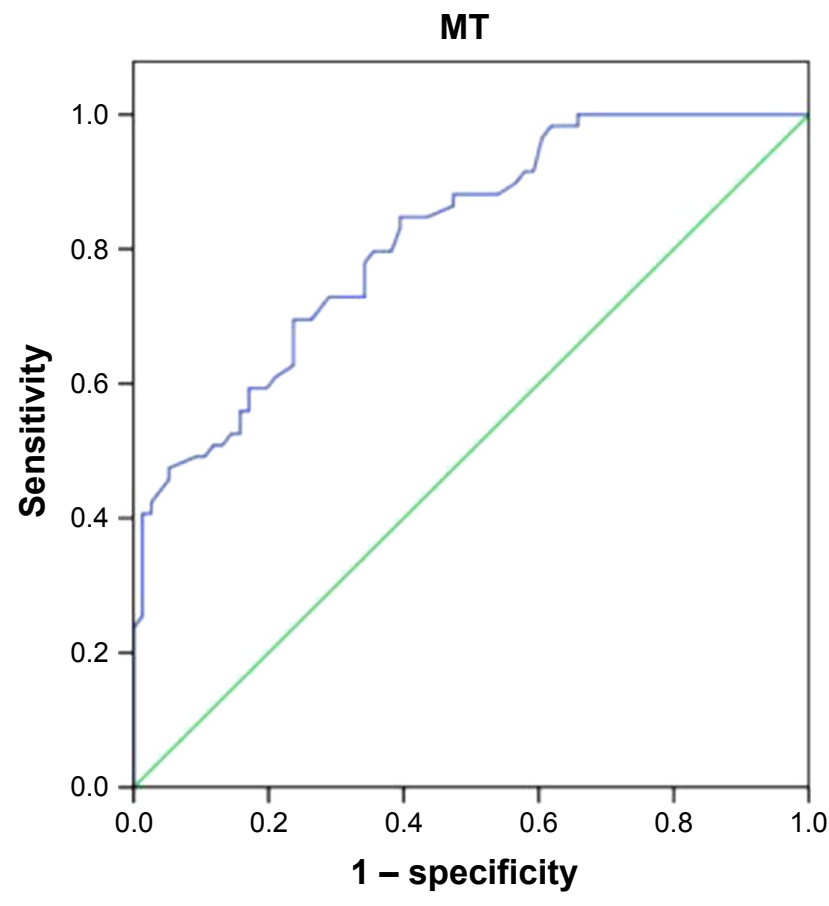

Figure $\mathbf{2}$ The result of ROC analysis of the gastrocnemius MT value in predicting low muscle mass.

Note: The tracings include ROC analysis of MT value in predicting low muscle mass (blue line) and baseline line (green line).

Abbreviations: MT, muscle thickness; ROC, receiver operating characteristic.

that the reproducibility of thickness measurements by ultrasonography was high $(r=0.96-0.99)$.

It is well known that body fat distribution and fat mass are different between men and women. However, there was no significant difference between men and women for muscle mass values estimated by US. ${ }^{13,28}$ One reason for these disparate results likely involves the fact that women have a larger proportion of muscle mass in their lower bodies. ${ }^{29}$ Another reason might be the age-related declines in androgen concentrations, which may be a strong etiologic factor for sarcopenia. ${ }^{30}$ Previous studies also showed significantly greater muscle mass in men compared to that in women at all ages and greater loss of muscle mass in men compared to that in women with increasing age..$^{29,31}$ The current study indicated that muscle mass was not significantly different between males and females. Although consensus statements from different regions of the world have suggested diagnostic criteria for males and females respectively, ROC analyses on the prediction of low muscle mass in this study included both males and females and the cutoff value of muscle mass thickness was $1.50 \mathrm{~cm}$. It had been reported that the cutoff value of muscle mass thickness for the prediction of sarcopenia was $1.69 \mathrm{~cm}$ for the right gastrocnemius muscle. ${ }^{26}$ The difference in the cutoff values for MT between these two studies might be caused by different classifications of 
participants (normal and low muscle mass, comparing sarcopenia and nonsarcopenia) and participants from different regions (comparing Asia and Europe).

\section{Limitations}

The potential limitations of this study include the low sample numbers and the fact that the subjects mainly came from the same region. Thus, the results of this study cannot be used to represent US measurements of the gastrocnemius muscle in a wide range of people. Moreover, this study lost 23 participants who were unable to perform the routine US position. These participants may suffer from severe sarcopenia. Thus, some potential participants with sarcopenia might be lost in this study. In addition, the loss of muscle mass in sarcopenia is a relatively slow process $-0.37 \mathrm{~kg} /$ year for the entire body. ${ }^{32}$ Thus, a comprehensive evaluation of the loss of muscle mass will require a study period of greater than 5 years.

\section{Conclusion}

Although DXA is the first choice for measuring muscle mass in patients with sarcopenia, DXA is not suitable for community screening. In this study, we have found the appropriate method to measure muscle mass - US measurement of gastrocnemius MT. This method is easy to master, with little deviation, and is convenient. According to the results of this study, the older people (including men and women) with gastrocnemius MT less than $1.5 \mathrm{~cm}$ can be considered as low muscle mass. It is hoped that US measurements would bring greater convenience for the diagnosis of sarcopenia in community screening.

\section{Disclosure}

The authors report no conflicts of interest in this work.

\section{References}

1. Delmonico MJ, Harris TB, Lee JS, et al. Alternative definitions of sarcopenia, lower extremity performance, and functional impairment with aging in older men and women. $J$ Am Geriatr Soc. 2007;55(5): 769-774.

2. Goodpaster BH, Park SW, Harris TB, et al. The loss of skeletal muscle strength, mass, and quality in older adults: the health, aging and body composition study. J Gerontol A Biol Sci Med Sci. 2006;61(10): 1059-1064.

3. Cruz-Jentoft AJ, Landi F, Topinková E, Michel JP. Understanding sarcopenia as a geriatric syndrome. Curr Opin Clin Nutr Metab Care. 2010;13(1):1-7.

4. Srikanthan P, Karlamangla AS. Relative muscle mass is inversely associated with insulin resistance and prediabetes. Findings from the third National Health and Nutrition Examination Survey. J Clin Endocrinol Metab. 2011;96(9):2898-2903.
5. Hsu YH, Liang CK, Chou MY, et al. Association of cognitive impairment, depressive symptoms and sarcopenia among healthy older men in the veterans retirement community in southern Taiwan: a cross-sectional study. Geriatr Gerontol Int. 2014;14(Suppl 1):102-108.

6. Chin SO, Rhee SY, Chon S, et al. Sarcopenia is independently associated with cardiovascular disease in older Korean adults: the Korea National Health and Nutrition Examination Survey (KNHANES) from 2009. PLoS One. 2013;8(3):e60119.

7. Cruz-Jentoft AJ, Baeyens JP, Bauer JM, et al. European Working Group on Sarcopenia in Older People: Sarcopenia: European consensus on definition and diagnosis: report of the European Working Group on sarcopenia in older people. Age Ageing. 2010;39:412-423.

8. Fielding RA, Vellas B, Evans WJ, et al. Sarcopenia: an undiagnosed condition in older adults. Current consensus definition: prevalence, etiology, and consequences. International working group on sarcopenia. J Am Med Dir Assoc. 2011;12(4):249-256.

9. Chen LK, Liu LK, Woo J, et al. Sarcopenia in Asia: consensus report of the Asian Working Group for Sarcopenia. J Am Med Dir Assoc. 2014; 15(2):95-101.

10. Mclean RR, Shardell MD, Alley DE, et al. Criteria for clinically relevant weakness and low lean mass and their longitudinal association with incident mobility impairment and mortality: the foundation for the National Institutes of Health (FNIH) sarcopenia project. $J$ Gerontol A Biol Sci Med Sci. 2014;69(5):576-583.

11. Morat T, Gilmore KJ, Rice CL. Neuromuscular function in different stages of sarcopenia. Exp Gerontol. 2016;81:28-36.

12. Kim J, Wang Z, Heymsfield SB, Baumgartner RN, Gallagher D. Totalbody skeletal muscle mass: estimation by a new dual-energy X-ray absorptiometry method. Am J Clin Nutr. 2002;76(2):378-383.

13. Sanada K, Kearns CF, Midorikawa T, Abe T. Prediction and validation of total and regional skeletal muscle mass by ultrasound in Japanese adults. Eur J Appl Physiol. 2006;96(1):24-31.

14. Bastiaanse LP, Hilgenkamp TI, Echteld MA, Evenhuis HM. Prevalence and associated factors of sarcopenia in older adults with intellectual disabilities. Res Dev Disabil. 2012;33(6):2004-2012.

15. Brodie DA. Techniques of measurement of body composition. Part II. Sports Med. 1988;5(2):74-98.

16. Jones PR, Davies PS, Norgan NG. Ultrasonic measurements of subcutaneous adipose tissue thickness in man. Am J Phys Anthropol. 1986; 71(3):359-363.

17. Abe T, Kondo M, Kawakami Y, Fukunaga T. Prediction equations for body composition of Japanese adults by B-mode ultrasound. Am J Hum Biol. 1994;6(2):161-170.

18. Ishida Y, Carroll JF, Pollock ML, Graves JE, Leggett SH. Reliability of B-mode ultrasound for the measurement of body fat and muscle thickness. Am J Hum Biol. 1992;4(4):511-520.

19. Strasser EM, Draskovits T, Praschak M, Quittan M, Graf A. Association between ultrasound measurements of muscle thickness, pennation angle, echogenicity and skeletal muscle strength in the elderly. Age. 2013;35(6):2377-2388.

20. Kaya A, Kara M, Tiftik T, et al. Ultrasonographic evaluation of the muscle architecture in patients with systemic lupus erythematosus. Clin Rheumatol. 2013;32(8):1155-1160.

21. Scanlon TC, Fragala MS, Stout JR, et al. Muscle architecture and strength: adaptations to short-term resistance training in older adults. Muscle Nerve. 2014;49(4):584-592.

22. Tsubahara A, Chino N, Akaboshi K, Okajima Y, Takahashi H. Age-related changes of water and fat content in muscles estimated by magnetic resonance (MR) imaging. Disabil Rehabil. 1995;17(6):298-304.

23. Frantzell A, Ingelmark BE. Occurrence and distribution of fat in human muscles at various age levels; a morphologic and roentgenologic investigation. Acta Soc Med Ups. 1951;56(1-2):59-87.

24. Watanabe Y, Yamada Y, Fukumoto Y, et al. Echo intensity obtained from ultrasonography images reflecting muscle strength in elderly men. Clin Interv Aging. 2013;8:993-998. 
25. Nijholt W, Scafoglieri A, Jager-Wittenaar H, Hobbelen JSM, van der Schans CP. The reliability and validity of ultrasound to quantify muscles in older adults: a systematic review. J Cachexia Sarcopenia Muscle. 2017;8(5):702-712.

26. Kuyumcu ME, Halil M, Kara Ö, et al. Ultrasonographic evaluation of the calf muscle mass and architecture in elderly patients with and without sarcopenia. Arch Gerontol Geriatr. 2016;65:218-224.

27. Chang KV, Wu WT, Huang KC, Jan WH, Han DS. Limb muscle quality and quantity in elderly adults with dynapenia but not sarcopenia: An ultrasound imaging study. Exp Gerontol. 2018;108:54-61.

28. Abe T, Kearns CF, Fukunaga T. Sex differences in whole body skeletal muscle mass measured by magnetic resonance imaging and its distribution in young Japanese adults. Br J Sports Med. 2003;37(5):436-440.
29. Gallagher D, Visser M, de Meersman RE, et al. Appendicular skeletal muscle mass: effects of age, gender, and ethnicity. J Appl Physiol. 1997; 83(1):229-239.

30. Morley JE. Hormones and the aging process. J Am Geriatr Soc. 2003; 51(7 Suppl):S333-S337.

31. Janssen I, Heymsfield SB, Wang ZM, Ross R. Skeletal muscle mass and distribution in 468 men and women aged 18-88 yr. J Appl Physiol. 2000;89(1):81-88.

32. Song MY, Ruts E, Kim J, Janumala I, Heymsfield S, Gallagher D. Sarcopenia and increased adipose tissue infiltration of muscle in elderly African American women. Am J Clin Nutr. 2004;79(5):874-880.
Clinical Interventions in Aging

\section{Publish your work in this journal}

Clinical Interventions in Aging is an international, peer-reviewed journal focusing on evidence-based reports on the value or lack thereof of treatments intended to prevent or delay the onset of maladaptive correlates of aging in human beings. This journal is indexed on PubMed Central, MedLine,

\section{Dovepress}

CAS, Scopus and the Elsevier Bibliographic databases. The manuscript management system is completely online and includes a very quick and fair peer-review system, which is all easy to use. Visit http://www.dovepress. $\mathrm{com} /$ testimonials.php to read real quotes from published authors. 